\title{
COLDDIAG: A Cold Vacuum Chamber for Diagnostics
}

\author{
Sara Casalbuoni, Tilo Baumbach, Stefan Gerstl, Andreas Grau, Michael Hagelstein, David Saez de Jauregui, \\ Cristian Boffo, Günther Sikler, Vincent Baglin, Roberto Cimino, Mario Commisso, Bruno Spataro, \\ Andrea Mostacci, Matthew Cox, Jos Schouten, Erik Wallèn, Ralf Weigel, James Clarke, Duncan Scott, \\ Tom Bradshaw, Ian Shinton, and Roger Jones
}

\begin{abstract}
One of the still open issues for the development of superconducting insertion devices is the understanding of the heat load induced by the beam passage. With the aim of measuring the beam heat load to a cold bore and in order to gain a deeper understanding in the beam heat load mechanisms, a cold vacuum chamber for diagnostics is under construction. We plan to have access with the same set-up to a number of different diagnostics, so we are implementing: i) retarding field analysers to measure the electron flux, ii) temperature sensors to measure the total heat load, iii) pressure gauges, iv) and mass spectrometers to measure the gas content. The inner vacuum chamber will be removable in order to test different geometries and materials. COLDDIAG is built to fit in a short straight section at ANKA, but we are proposing its installation in different synchrotron light sources with different energies and beam characteristics. A first installation in DIAMOND is planned in June 2011. Here we describe the technical design report of this device and the planned measurements with beam.
\end{abstract}

Index Terms-Accelerator magnets, heating, undulator.

\section{INTRODUCTION}

$\mathbf{S}$ UPERCONDUCTING insertion devices (IDs) have higher fields for a given gap and period length compared with the state of the art technology of permanent magnet IDs. This

Manuscript received August 01, 2010; accepted November 23, 2010. Date of publication January 06, 2011; date of current version May 27, 2011.

S. Casalbuoni, T. Baumbach, S. Gerstl, A. Grau, M. Hagelstein, and D. Saez de Jauregui are with the Karlsruhe Institute of Technology, Hermann-von-Helmholtz-Platz 1, Karlsruhe 76344, Germany (e-mail: sara. casalbuoni@kit.edu).

C. Boffo and G. Sikler are with Babcock Noell GmbH, Alfred-Nobel-Str 20, Würzburg 97080, Germany.

V. Baglin is with the European Organization for Nuclear Research CERN,CH-1211, Genève 23, Switzerland.

M. Cox and J. Schouten are with Diamond Light Source, Didcot, Oxfordshire, OX11 ODE, U.K.

R. Cimino, M. Commisso, and B. Spataro are with the Laboratori Nazionali di Frascati, Via E. Fermi 40, Frascati 00044 (Roma), Italy.

A. Mostacci is with Università degli Studi di Roma "La Sapienza," Piazzale Aldo Moro 5, Roma 00185, Italy.

E. J. Wallén is with Lund University MAX-lab, SE-221 00 Lund, Sweden.

R. Weigel is with the Max Planck Institute for Metals Research, Heisenbergstrasse 3, Stuttgart D-70569, Germany.

J. Clarke and D. Scott are with the STFC Daresbury Laboratory, Daresbury, Warrington, Cheshire, WA4 4AD, U.K.

T. Bradshaw is with the STFC Rutherford Appleton Laboratory, Harwell Science and Innovation Campus, Didcot, OX11 0QX, U.K.

R. Jones and R. Shinton are with the University of Manchester, Oxford Road, Manchester M13 9PL, U.K., and with the Cockcroft Institute of Accelerator Science and Technology, Daresbury, WA4 4AD, U.K.

Color versions of one or more of the figures in this paper are available online at http://ieeexplore.ieee.org.

Digital Object Identifier 10.1109/TASC.2010.2096176 technological solution is very interesting for synchrotron light sources since it permits to increase the brilliance and/or the photon energy at moderate costs. One of the key issues for the development of superconducting IDs is the understanding of the beam heat load to the cold vacuum chamber. The beam heat load is a key parameter to determine the cryogenic design and the choice of the superconducting material for future superconducting IDs. Possible beam heat load sources are: synchrotron radiation, resistive wall heating, electron and/or ion bombardment and RF effects. The values of the beam heat load due to synchrotron radiation and resistive wall heating have been calculated and compared for the cold bores installed at different light sources with the measured values. The disagreement between beam heat load measured and calculated is not understood [1]-[3].

Studies performed with the cold bore superconducting undulator installed at the synchrotron radiation source ANKA suggest that the main contribution to the beam heat load is due to secondary electron bombardment. The electron bombardment model appears to be consistent with the beam heat load and the dynamic pressure rise observed for bunch lengths of about $10 \mathrm{~mm}$ [2]. Low energy electrons (few eV) are accelerated by the electric field of the beam towards the wall of the vacuum chamber, introducing heat to the cold liner and causing nonthermal desorption of gas from the cryogenic surface.

It has been shown [4] that in order to reproduce the pressure measurements in the ANKA cold bore superconducting undulator, it is necessary to include in the equations of gas dynamic balance, electron stimulated desorption with a shorter decay than the beam lifetime. This implies a very fast avalanche like growth of the electron flux as a function of beam current, suggesting electron multipacting. A common cause of electron bombardment is the buildup of an electron cloud, which strongly depends on the chamber surface properties. The surface properties as secondary electron yield, photoemission yield, photoemission induced electron energy distribution, needed in the simulation codes to determine the eventual occurrence and size of an electron cloud buildup, have only partly been measured for a cryosorbed gas layer. Even using uncommonly large values for these parameters, the heat load inferred from the ECLOUD simulations [5] is about one order of magnitude lower than the measurements [6]. Mechanisms different than the classical buildup of the electron cloud that can explain the electron bombardment and multipacting are currently under study at KIT.

In order to gain a quantitative understanding of the beam heat load and to find effective remedies we have designed a cold vacuum chamber for diagnostics (see [3] and Fig. 1) to be in- 


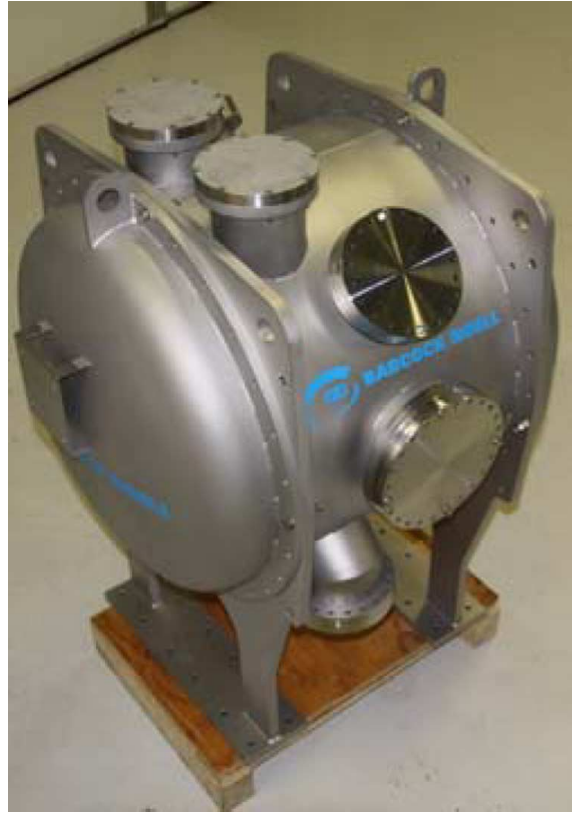

Fig. 1. The cryostat.

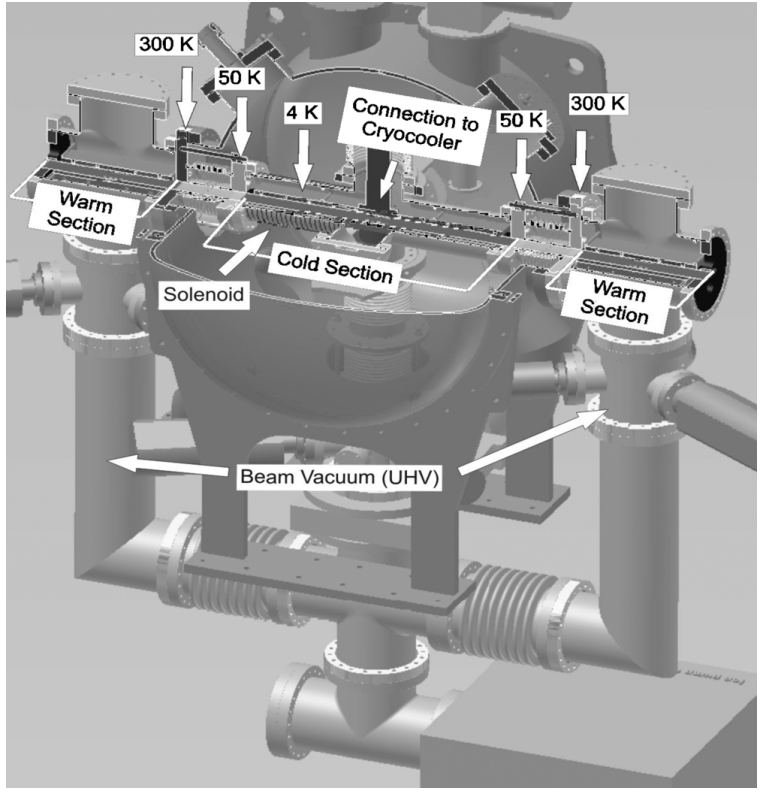

Fig. 2. Sketch of the cryostat: the two warm sections and the cold section are shown, as well as the UHV vacuum separated by the isolation vacuum by a 6-way cross.

stalled in synchrotron light sources. The goal is to measure the heat load, the pressure, the gas content and the flux and spectrum of the low energy electrons bombarding the wall.

A first installation in the Diamond Light Source is foreseen for June 2011. In the following we describe the technical design report of this device and the planned measurements with beam.

\section{THE VACUUM CHAMBER}

COLDDIAG consists of a cold vacuum chamber located between two warm sections (see Fig. 2), one upstream and one
TABLE I

THERMAL LOAD BUDGET WITHOUT BEAM

\begin{tabular}{lcc}
\hline \hline \multicolumn{1}{c}{ Cold } & \multicolumn{1}{c}{ mass } & Shield \\
\hline Radiation $[W]$ & 0.2 & 12 \\
Conduction $[W]$ & 0.55 & 36 \\
Sum $[W]$ & $\mathbf{0 . 7 5}$ & $\mathbf{4 8}$
\end{tabular}

Cryocooler power $[\mathrm{W}] \quad 1.5 \mathrm{~W} @ 4.2 \mathrm{~K} \quad 50 \mathrm{~W} @ 60 \mathrm{~K}$

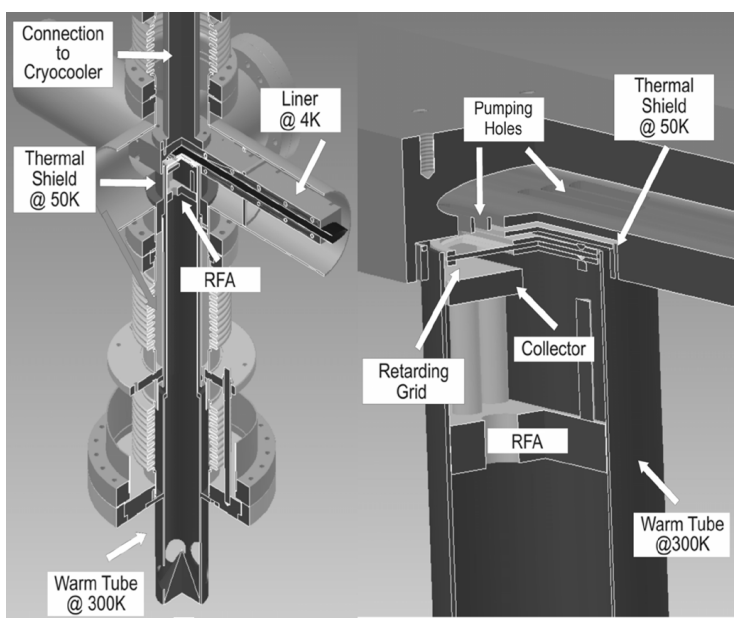

Fig. 3. Sketch of the diagnostic port in the cold section: the diagnostics devices are connected to the cold liner trough a warm tube to avoid gas condensation along the path between liner and diagnostic devices.

downstream. This will allow us to observe the influence of synchrotron radiation on the beam heat load and to make a direct comparison of the cryogenic and room temperature regions, with and without cryosorbed gas layer, respectively.

The electron beam will go through a liner designed to be exchangeable. The first liner that will be tested at DIAMOND is machined from solid copper (also used for thermal stabilisation) which is itself plated with $30 \mu \mathrm{m}$ of pure copper. The resistance at low temperatures in the first few $\mu \mathrm{m}$ of material exposed to the beam is an important parameter that determines resistive wall heating losses. The $30 \mu \mathrm{m}$ of copper plating resembles the surface seen by the electron beam in the liner used in the ANKA superconducting undulators, made of $300 \mu \mathrm{m}$ stainless steel foil with $30 \mu \mathrm{m}$ of copper plating [3], [7].

The vacuum system of COLDDIAG consists of two volumes: the isolation vacuum and the beam vacuum (UHV). A DN100 CF stainless steel 6-way cross separates the two vacua (Fig. 2). The beam vacuum, shown in Fig. 2, includes all the diagnostic devices as well as the liner. This volume will be pumped with a $500 \mathrm{l} / \mathrm{s}$ ion pump. In addition during cold operation the cold bore of the chamber works like a cryopump. A base pressure of about $10^{-11} \mathrm{mbar}$ is expected in the UHV vacuum in absence of the beam. The usage of bellows allows for the thermal shrinkage of the cold parts as they cool down. The bellows are optimized to minimize the heat transfer. They are also equipped with RF-fingers.

In order to simulate the cold bore of superconducting insertion devices the liner must be cooled down to reach about $4 \mathrm{~K}$ in absence of beam, and there must be the possibility to operate at 


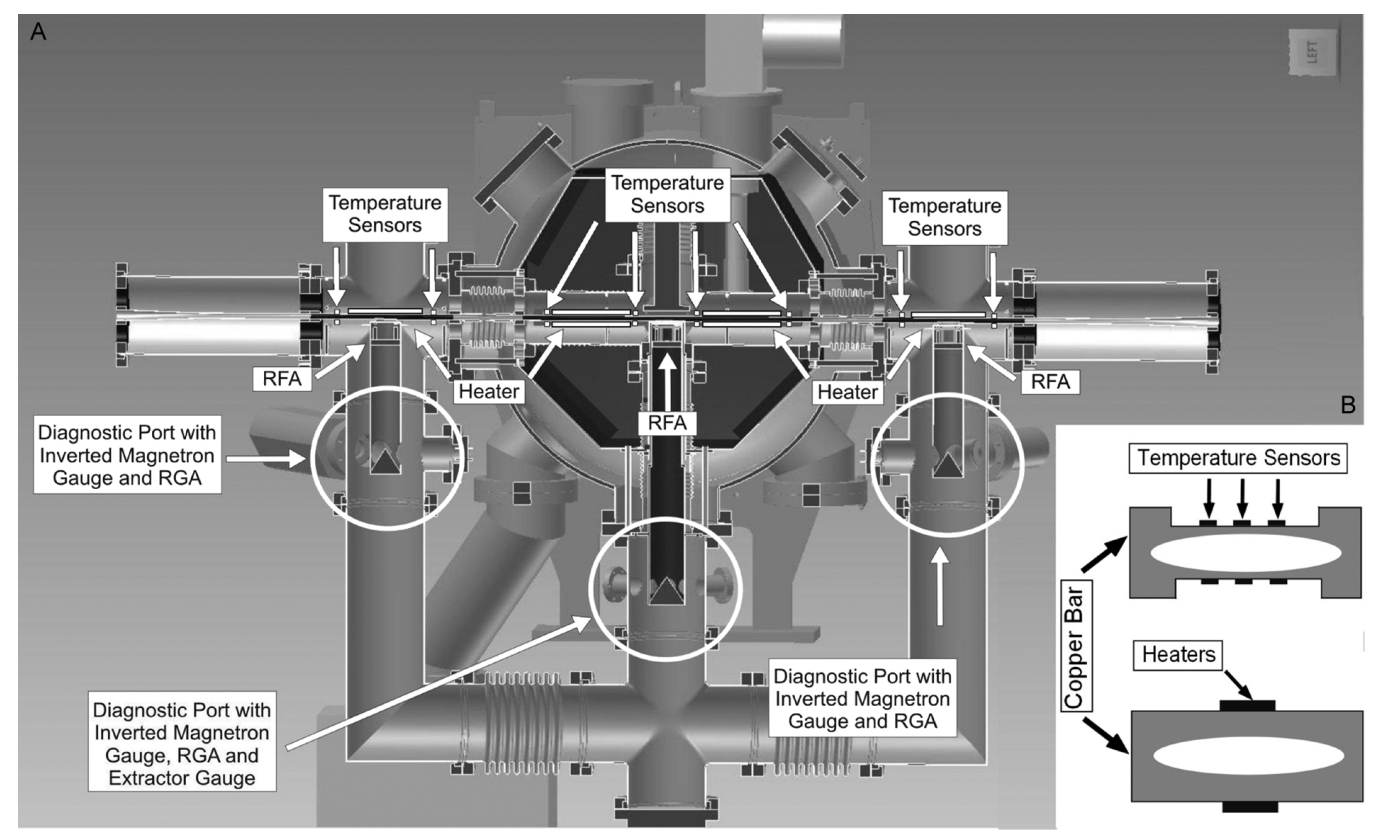

Fig. 4. Overview of the diagnostics installed in the cold and in the two warm sections of COLDDIAG: retarding field analyser, residual gas analyser, pressure gauge, temperature sensors, and heaters to simulate the heating from the beam.

higher temperatures up to $300 \mathrm{~K}$. COLDDIAG is cryogen-free and cooled by a Sumitomo RDK-415D cryocooler. The system has 3 temperature regimes: $300 \mathrm{~K}, 60 \mathrm{~K}$ at the radiation shield, and $4 \mathrm{~K}$ at the liner. Calculations of thermal radiation and conductance performed by Babcock Noell, show a heat load of 48 $\mathrm{W}$ on the shield at $60 \mathrm{~K}$, and $0.75 \mathrm{~W}$ on the cold liner at $4 \mathrm{~K}$ (see Table I). With a maximum cooling power of $50 \mathrm{~W}$ at $60 \mathrm{~K}$ at the 1 st stage of the cryocooler and $1.5 \mathrm{~W}$ at $4.2 \mathrm{~K}$ at the $2 \mathrm{nd}$ stage one cryocooler is anticipated to be enough to obtain $4 \mathrm{~K}$ on the liner in absence of beam.

In order to suppress the low energy electrons bombarding the wall, a solenoid on the beam axis producing a maximum field of 100 Gauss will be wound around on one of the long arms of the cold UHV cross (symbolized by the spiral in Fig. 2). For the diagnostic port in the cold section a design based on the COLDEX device [8] was chosen.

The diagnostic devices are connected to the cold liner through a warm tube to avoid gas condensation along the path between liner and diagnostic devices (see Fig. 3).

We are interested primarily in using COLDDIAG to study the influence of the beam on a straight liner, and unwanted effects from steps, pumping and diagnostic ports must be minimized. These must be reduced to below about $0.5 \mathrm{~W}$ so as not to heat the liner above about $4 \mathrm{~K}$ (see Table I). Numerical simulations on the current design are being made at the University of Manchester to calculate these effects.

\section{DiAgnOSTICS}

The following diagnostics will be implemented: temperature sensors to measure the total heat load, retarding field analysers to measure the electron flux, pressure gauges, and mass spectrometers to measure the gas composition.

In total 38 temperature sensors will allow us to monitor the status of the chamber and measure the beam heat load. Eight calibrated Hereaus C220 PT100 sensors in each of the warm

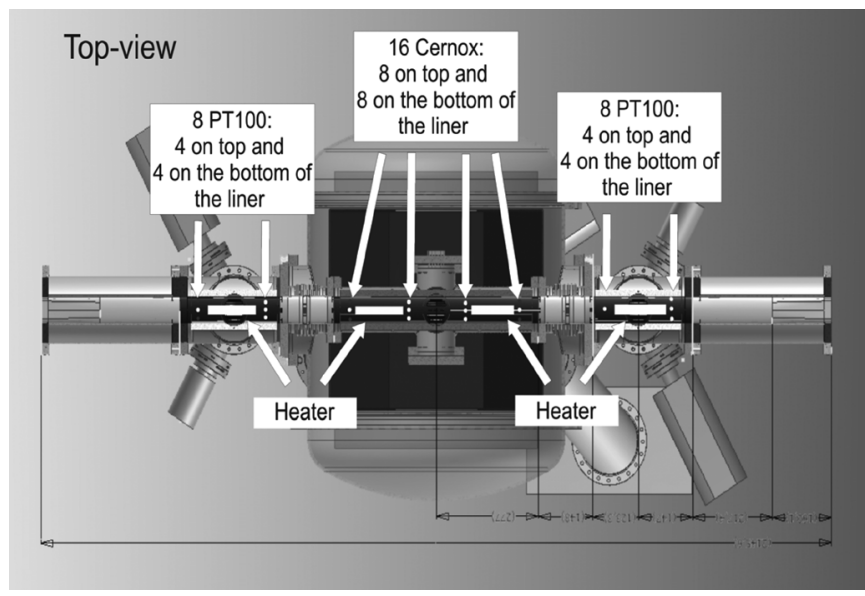

Fig. 5. Top-view of COLDDIAG showing the location of the temperature sensors and heaters along the liner. On the bottom part of the liner the same geometrical distribution of heaters and sensors will be used.

sections and 16 calibrated Lakeshore Cernox 1050-SD in the cold section are placed directly on the liner (see Fig. 4) by spring loaded screws. This gives us the possibility to obtain not only the beam heat load but also the heat distribution on the liner. To calibrate the temperature sensors to the beam heat load we use stainless steel heating bands, which will be placed on the outer part of the copper bars, to simulate the heating from the beam (see Figs. 4 and 5).

In each of the connection pipes, between the liner and the diagnostic ports a small half moon shaped retarding field analyser (RFA) will be placed to obtain the electron flux of the electrons impinging the wall. During calibration of a similar RFA for the ANKA storage ring at INFN in Frascati it turned out, that it is not possible to obtain the electron distribution with the current setup [9]. This might be due to a background of secondary electrons produced inside the RFA. At the moment we are testing an 
improved setup using a lock-in technique. To solve the problem of the secondary electrons produced in the RFA we use an AC voltage inductively coupled to the retarding grid and detect the signal on the collector plate. With this setup we can directly acquire the first derivative of the electron current on the collector, which gives us the electron energy distribution.

At each of the three diagnostic ports, an inverted magnetron gauge (minimal measurable pressure $10^{-10}$ mbar) and a residual gas analyser will be installed, to monitor the total pressure and the gas composition of the beam vacuum. The middle diagnostic port will also be equipped with an extractor gauge, which is more sensitive than the inverted magnetron gauges (minimal measurable pressure $10^{-11} \mathrm{mbar}$ ). The pressure gauges and mass spectrometers will be calibrated at CERN.

Through the middle diagnostic port also it will be possible to inject different gases. Therefore a heated high precision all metal leak valve, which allows to control the gas flow to the chamber down to $10^{-10} \mathrm{mbar} \mathrm{l} / \mathrm{s}$ was chosen. To study the effect of the injected gases under controlled conditions a defined amount of molecules must be homogeneously deposited on the cold liner foil. To do so we plan to warm up the liner with the installed heaters to about $150 \mathrm{~K}$. Tests will be done to verify that this is warm enough to desorb the relevant gases. This will clean the liner surface as the boiling point of the most gases in the storage ring is below this temperature. Together with the cool down of the liner the gas will be injected until cryopumping of the cold surface starts. At this point, which will show up as a dramatic pressure decrease, the gas injection will be stopped. In several offline tests the amount of deposited gas will be checked by warming up the liner again and measuring the pressure increase of the adsorbed gas with the RGA's.

\section{Planned Measurements}

During normal user operation the temperature, electron flux, pressure and gas composition will be monitored to collect statistics. During machine physics at Diamond we plan to change:

1) the average beam current to compare the beam heat load data with synchrotron radiation and resistive wall heating predictions,
2) the bunch length to compare with resistive wall heating predictions,

3) the filling pattern in particular the bunch spacing to test the relevance of the electron cloud as heating mechanisms,

4) beam position to test the relevance of synchrotron radiation and the gap dependence of the beam heat load,

5 ) inject different gases naturally present in the beam vacuum $\left(\mathrm{H}_{2}, \mathrm{CO}, \mathrm{CO}_{2}, \mathrm{CH}_{4}\right)$ to understand the influence of the cryosorbed gas layer on the beam heat load and eventually identify the gases to be reduced in the beam vacuum.

\section{SUMMARY}

In this paper we reported about the design and the foreseen diagnostic devices of COLDDIAG. The factory acceptance test is planned by the end of the summer of 2010, first tests of the experimental setup focused on gas injection will take place at ANKA out of the storage ring from October 2010 and February 2011. A first installation of COLDDIAG in an electron storage ring is planned for June 2011 in the Diamond Light Source.

\section{REFERENCES}

[1] E. Wallèn and G. LeBlanc, Cryogenics, vol. 44, p. 879, 2004.

[2] S. Casalbuoni, A. Grau, M. Hagelstein, R. Rossmanith, F. Zimmermann, B. Kostka, E. Mashkina, E. Steffens, A. Bernhard, D. Wollmann, and T. Baumbach, Phys. Rev. ST Accel. Beams, vol. 10, p. 093202, 2007.

[3] S. Casalbuoni, T. Baumbach, A. Grau, M. Hagelstein, R. Rossmanith, V. Baglin, B. Jenninger, R. Cimino, M. Cox, E. Mashkina, and E. Wallèn, in Proceedings of the 11th European Particle Accelerator Conference, Genoa, 2008, Genoa, Italy, 2008, EPS-AG.

[4] S. Casalbuoni, S. Schleede, D. Saez de Jauregui, M. Hagelstein, and P. F. Tavares, Phys. Rev. ST Accel. Beams, vol. 13, p. 073201, 2010.

[5] G. Rumolo and F. Zimmermann, Report No. CERN SLNote-2002-016.

[6] U. Iriso, S. Casalbuoni, G. Rumolo, and F. Zimmermann, in Proceedings of Particle Accelerator Conference 09, Vancouver, Canada [Online]. Available: http://trshare.triumf.ca/ pac09proc/Proceedings/

[7] C. Boffo, W. Walter, T. Baumbach, S. Casalbuoni, S. Gerstl, A. Grau, M. Hagelstein, and D. Saez de Jauregui, This Proceedings.

[8] V. Baglin, I. R. Collins, and B. Jenninger, Vacuum, vol. 73, pp. 201-206, 2004

[9] D. Saez de Jauregui, S. Casalbuoni, A. Grau, M. Hagelstein, R. Cimino, M. Commisso, E. Mashkina, and R. Weigel, in Proceedings of Particle Accelerator Conference 09, Vancouver, Canada [Online]. Available: http://trshare.triumf.ca/ pac09proc/Proceedings/ 